\title{
Narcotic Bowel Syndrome: Is It More Common Than Before?
}

TO THE EDITOR: We read the recent article Farmer et $\mathrm{al}^{1}$ on narcotic bowel syndrome (NBS) with great interest. The authors have comprehensively described a case of NBS along with the exciting pathophysiology. It further illustrates the common vicious cycle we see in daily clinical practice: a patient with chronic abdominal pain being prescribed opiates, which further worsens the pain and thereby escalating opiate dosage which produces a paradoxical effect. We would like to share some comments on the article.

Previous reports have shown that approximately $6 \%$ of patients taking narcotics will develop NBS chronically. ${ }^{2}$ However, due to increased recognition of these cases, it might be more common than previously thought. The success of the detoxification regimen used for NBS could be short term as we have seen in our patient population. Though patients respond significantly in the initial weeks, most of the patients experience recurrent symptoms in a span of 2-3 months leading to a high recidivism rate. Furthermore, the authors mention that constipation may develop with opiate withdrawal and laxatives maybe used in the absence of an obstruction. However, it is usually diarrhea that is predominant in these patients in the acute phase of opioid withdrawal. ${ }^{3}$ In addition, when patients on chronic opioids present with severe constipation, abdominal discomfort and post-prandial emesis, the possibility of generalized gastroparesis should be in the differential for the work up of these patients. ${ }^{4}$ A trial of prokinetic drugs could be helpful in this subset of population before the use of selective opioid antagonist such as methylnaltrexone.

Palaniappan Manickam and Abdulhassan Saad Gastroenterology Division, Department of Internal Medicine, William Beaumont Hospital, Royal Oak, MI, USA

1. Farmer AD, Ferdinand E, Aziz Q. Opioids and the gastrointestinal tract - a case of narcotic bowel syndrome and literature review. J Neurogastroenterol Motil 2013;19:94-98.

2. Drossman DA, Morris CB, Edwards H, et al. Diagnosis, characterization, and 3-month outcome after detoxification of 39 patients with narcotic bowel syndrome. Am J Gastroenterol 2012;107:1426-1440.

3. Kosten TR, O'Connor PG. Management of drug and alcohol withdrawal. N Eng J Med 2003;348:1786-1795.

4. Parkman HP, Hasler WL, Fisher RS; American Gastroenterological Association. American Gastroenterological Association technical review on the diagnosis and treatment of gastroparesis. Gastroenterology 2004;127:1592-1622.

\section{Conflicts of interest: None.}

